\title{
Anomalous Hall effect at the spontaneously electron-doped polar surface of $\mathrm{PdCoO}_{2}$ ultrathin films
}

\author{
T. Harada $\odot,{ }^{1, *}$ K. Sugawara,${ }^{2,3,4}$ K. Fujiwara, ${ }^{1}$ M. Kitamura, ${ }^{5}$ S. Ito, ${ }^{1}$ T. Nojima, ${ }^{1}$ K. Horiba,${ }^{5}$ H. Kumigashira, ${ }^{5,6}$ \\ T. Takahashi, ${ }^{2,3,4}$ T. Sato, ${ }^{2,3,4}$ and A. Tsukazaki ${ }^{1,4}$ \\ ${ }^{1}$ Institute for Materials Research, Tohoku University, Sendai, Japan \\ ${ }^{2}$ Department of Physics, Tohoku University, Sendai, Japan \\ ${ }^{3}$ WPI Advanced Institute for Materials Research, Tohoku University, Sendai, Japan \\ ${ }^{4}$ Center for Spintronics Research Network, Tohoku University, Sendai, Japan \\ ${ }^{5}$ Photon Factory, Institute of Materials Structure Science, High Energy Accelerator Research Organization, Tsukuba, Ibaraki, Japan \\ ${ }^{6}$ Institute of Multidisciplinary Research for Advanced Materials, Tohoku University, Sendai, Japan
}

(Received 19 August 2019; revised manuscript received 10 February 2020; accepted 19 February 2020; published 9 March 2020)

\begin{abstract}
We revealed the electrical transport through surface ferromagnetic states of a nonmagnetic metal $\mathrm{PdCoO}_{2}$. Electronic reconstruction at the Pd-terminated surface of $\mathrm{PdCoO}_{2}$ induces Stoner-like ferromagnetic states, which could lead to spin-related phenomena among the highly conducting electrons in $\mathrm{PdCoO}_{2}$. Fabricating a series of nanometer-thick $\mathrm{PdCoO}_{2}$ thin films, we detected a surface-magnetization-driven anomalous Hall effect via systematic thickness- and termination-dependent measurements. Besides, we discuss that finite magnetic moments in electron doped $\mathrm{CoO}_{2}$ triangular lattices may have given rise to additional unconventional Hall resistance.
\end{abstract}

DOI: 10.1103/PhysRevResearch.2.013282

Layered transition-metal oxides have given great insight into the role of dimensionality in correlated electron physics [1-4]. Among the many different oxides, the delafossite metal $\mathrm{PdCoO}_{2}$ is a unique system in which monovalent $\mathrm{Pd}^{+}$ions are stabilized in the form of a two-dimensional (2D) Pd sheet that is sandwiched by $\left[\mathrm{CoO}_{2}\right]^{-}$layers [5-7] [Fig. 1(a)]. This $2 \mathrm{D} \mathrm{Pd}^{+}$sheet [Fig. 1(b)] provides a highly dispersive $4 d$ dominant conduction band [8-11], while $\left[\mathrm{CoO}_{2}\right]^{-}$forms an insulating block layer [Fig. 1(c)]. $\mathrm{PdCoO}_{2}$ bulk single crystals exhibit highly anisotropic electrical conduction, with the electrical conductivity exceeding that of elemental Pd [6]. More surprisingly, the long mean free path of the interacting dense electrons $(\sim 20 \mu \mathrm{m})$ induces hydrodynamic collective electron motion, as observed in high-mobility systems [12-16].

Because of the ionicity of the $\mathrm{Pd}^{+}$and $\left[\mathrm{CoO}_{2}\right]^{-}$layers [Fig. 1(a)], spontaneous charge compensation is induced at the polar surface. The electronic structure of the surface significantly differs from that of the bulk, and shows a strong termination-dependence. In particular, surface ferromagnetic state emerges at the Pd-terminated surfaces [17]. The surface charge compensation introduces extra electrons into the $\mathrm{Pd}$ surface layer, and this increase in electron density shifts the surface-Pd energy band to a higher binding energy relative to the original bulk energy band. This results in a flat branch of the surface band occurring near the Fermi level $\left(E_{\mathrm{F}}\right)$. The high

\footnotetext{
*Corresponding author: t.harada@imr.tohoku.ac.jp

Published by the American Physical Society under the terms of the Creative Commons Attribution 4.0 International license. Further distribution of this work must maintain attribution to the author(s) and the published article's title, journal citation, and DOI.
}

density of correlated electrons in the flat band increases the Stoner instability, resulting in the formation of spin-split surface Pd bands [17] [Fig. 1(d)]. This causes the Pd-terminated surface to become ferromagnetic, even though bulk $\mathrm{PdCoO}_{2}$ is nonmagnetic [17].

Indeed, Stoner-like splitting has been detected in the Pdterminated surface of $\mathrm{PdCoO}_{2}$ bulk single crystals using angle-resolved photoemission spectroscopy (ARPES) [18]. This observation suggested that a polar surface that contained highly conducting and spin-polarized electrons existed, which may lead to exotic spin-related transport phenomena in nonmagnetic $\mathrm{PdCoO}_{2}$. However, it is difficult to probe this type of surface transport in bulk samples because current shunting by the vast bulk volume obscures the surface conduction. To overcome this, here we examine ultrathin $\mathrm{PdCoO}_{2}$ films. By altering film thickness [19], we detected the signal from spinrelated transport in these ultrathin films, demonstrating that the surface ferromagnetism occurred within a limited thickness $d_{s}$ near the surface (Fig. 1(e) and Fig. S1 in Ref. [20]).

The preparation of Pd-terminated surfaces is essential to induce surface ferromagnetism via Stoner splitting [17]. We adopted a thin-film approach [19] in this study, as opposed to the bulk cleavage method. We fabricated nanometer-thick $c$-axis-oriented $\mathrm{PdCoO}_{2}$ films on $\mathrm{Al}_{2} \mathrm{O}_{3}$ (0001) $\left(c-\mathrm{Al}_{2} \mathrm{O}_{3}\right)$ substrates using pulsed laser deposition (Fig. S2 [20]). A typical, high-angle annular dark-field scanning transmission electron microscope (HAADF-STEM) image around the filmsubstrate interface is shown in Fig. 1(f). The bright and dark layers clearly alternated along the normal to the film plane, which correspond to the $\mathrm{Pd}^{+}$and $\left[\mathrm{CoO}_{2}\right]^{-}$layers, respectively. Because the initial growth layer was $\left[\mathrm{CoO}_{2}\right]^{-}$, and to ensure charge neutrality of the whole thin film, the final layer (top surface) was likely to be $\mathrm{Pd}^{+}$, although this could 

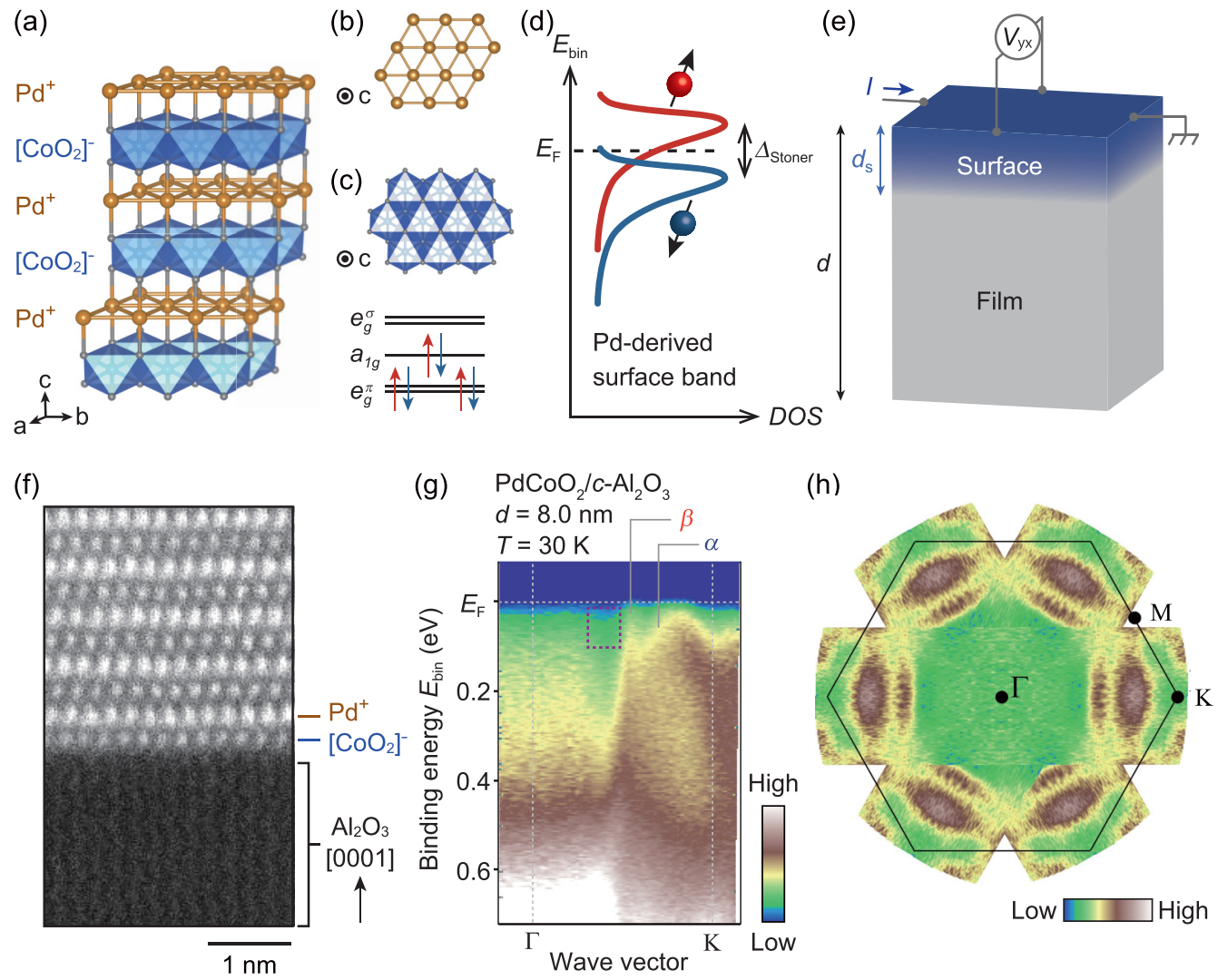

FIG. 1. Crystal structure and electronic structure of $\mathrm{PdCoO}_{2}$. (a) Crystal structure of $\mathrm{PdCoO}_{2}$. (b) Two-dimensional $\mathrm{Pd}$ layer. (c) $\mathrm{CoO} 2$ sublattice (top) and $\mathrm{Co}^{3+}$ spin arrangement (bottom). (d) The density of states (DOS) of surface Pd bands split by $\Delta_{\text {Stoner }}$ by Stoner instability $[17,18]$. (e) Schematic illustration of a $\mathrm{PdCoO}_{2}$ thin film with a surface ferromagnetic state extending over $d_{\mathrm{s}}$. Hall effect measurement probes the conduction throughout the entire film. (f) HAADF-STEM image of a $\mathrm{PdCoO}_{2}$ thin film on $\mathrm{Al}_{2} \mathrm{O}_{3}(0001)$, projected along the $\mathrm{Al}_{2} \mathrm{O}_{3}$ [ $\overline{1} 100$ ] direction. $\mathrm{Pd}$ and $\mathrm{CoO}_{2}$ sublattices can be seen on the $\mathrm{Al}_{2} \mathrm{O}_{3}$ substrate. $(\mathrm{g})$ The electronic band dispersion along the $\Gamma-K$ cut of a PdCoO film with $d=8.0 \mathrm{~nm}$. The spin-split surface Pd bands are shown as $\alpha$ and $\beta$. The purple dotted square indicates the region where the Co-derived surface band is expected to appear if it existed [18,22]. (h) Fermi surface of $\mathrm{PdCoO}_{2}$ obtained by symmetrizing the ARPES data in (g).

not be resolved in HAADF-STEM image. The electronic band dispersion at the film surface was characterized directly using ARPES as a surface-sensitive probe $[18,21,22]$. A pair of highly dispersive bands (denoted as $\alpha$ and $\beta$ ) were observed along the $\Gamma-K$ cut $[$ Fig. $1(\mathrm{~g})]$, which was consistent with Pd $4 d$-derived states [18]. The fact that there were no traces of Co-derived holelike states (region within the dotted purple square in Fig. 1(g)), combined with the Fermi surface map [Fig. 1(h)], was consistent with the Pd-terminated surface that was observed for the bulk single crystal [18]. As such, the ARPES spectra indicated that the final layer in our $\mathrm{PdCoO}_{2}$ films was predominately Pd. Furthermore, the nondegenerated $\alpha$ and $\beta$ bands and the flat branch of the $\alpha$ band located just below $E_{\mathrm{F}}[$ Fig. $1(\mathrm{~g})]$ are all hallmarks of Stoner-like splitting [18].

The Hall resistance $\left(R_{\mathrm{H}}\right)$ versus magnetic field $\left(\mu_{0} H\right)$ curve for a $13-\mathrm{nm}$-thick $\mathrm{PdCoO}_{2}$ film at $2 \mathrm{~K}$ is shown in Fig. 2(a). The $R_{\mathrm{H}}$ curves were obtained by subtracting the $\mu_{0} \mathrm{H}$-linear ordinary term, determined by the high-field data $\left(\mu_{0} H=5-7 \mathrm{~T}\right)$, from the measured transverse resistance $\left(R_{y x}=V_{y x} / I_{x}\right)$ (Fig. S3 [20]). While only a small nonlinear signal was observed from the 13-nm-thick sample [Fig. 2(a)], the signal became more pronounced as the thickness $(d)$ of the $\mathrm{PdCoO}_{2}$ film was decreased, as shown in Figs. 2(b) and 2(c). The $R_{\mathrm{H}}$ was independent of bias current ( $\left.I=1-100 \mu \mathrm{A}\right)$ used for the measurement of the mm-sized samples (Fig. S4 [20]). The comparable ordinary Hall coefficients from the three samples (the linear gradient at $\mu_{0} H=5-7 \mathrm{~T}$ in Figs. S3(a)-S3(c) [20]) indicated that the large $R_{\mathrm{H}}$ was induced by a reduction in $d$, rather than change in the thin-film quality.

A log-log plot of the sheet resistance under zero field $\left(R_{\text {sheet }}\right)$ as a function of $d$ is shown in Fig. 2(d). Fitting this plot yielded $d \log _{10}\left(R_{\text {sheet }}\right) / d \log _{10}(d)$ of $-1 \pm 0.2$ [blue line in Fig. 2(d)], and so $R_{\text {sheet }}$ was inversely proportional to $d$. Therefore the three-dimensional electrical conductivity $\left(\mathrm{S} \mathrm{cm}^{-1}\right)$ was constant, which secured that the quality of the films was unchanged down to $d=\sim 3 \mathrm{~nm}$. In contrast, fitting the plot of $R_{\mathrm{H}}$ at $9 \mathrm{~T}\left(R_{\mathrm{H}}{ }^{9 \mathrm{~T}}\right)$ versus $d$ [Fig. 2(e)] resulted in $d \log _{10}\left(R_{\mathrm{H}}{ }^{9 \mathrm{~T}}\right) / d \log _{10}(d)$ of $-2 \pm 0.3$ [red line in Fig. 2(e)]. The inverse square dependence of $R_{\mathrm{H}}{ }^{9 \mathrm{~T}}$ on $d\left(d^{-2}\right)$ can be understood by assuming that only the finite region near the surface was generating the nonlinear $R_{\mathrm{H}}$ [20]. This was consistent with the surface charge compensation model [17].

The magnetism of the $\mathrm{PdCoO}_{2}$ ultrathin films was examined using a superconducting quantum interference device. The saturation magnetization at $7 \mathrm{~T}\left(M_{\mathrm{s}}{ }^{7 \mathrm{~T}}\right)$ was plotted against temperature, as shown in Fig. 2(f). The $M_{\mathrm{s}}^{7 \mathrm{~T}}$ was estimated from the magnetization versus $\mu_{0} H$ curves by subtracting 

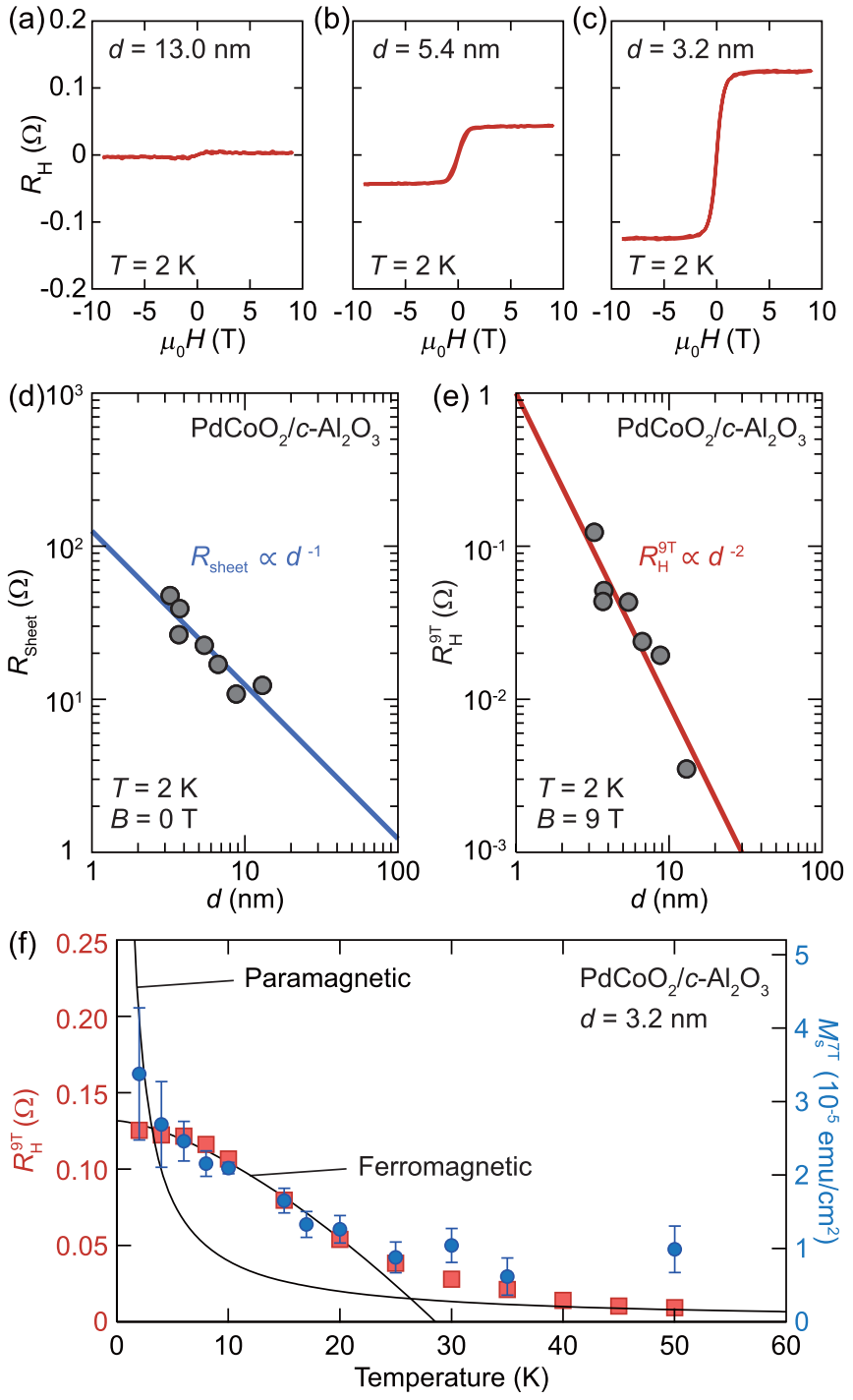

FIG. 2. AHE of $\mathrm{PdCoO}_{2}$ thin films. (a)-(c) $R_{\mathrm{H}}-\mu_{0} H$ curves of $\mathrm{PdCoO}_{2}$ films measured at $2 \mathrm{~K}$ with different thicknesses $(d)(d=$ 13.0, 5.4, and $3.2 \mathrm{~nm}$, respectively). (d) Sheet resistance $\left(R_{\text {sheet }}\right)$ at $B=0 \mathrm{~T}$ and $(\mathrm{e}) R_{\mathrm{H}}$ at $B=9 \mathrm{~T}\left(R_{\mathrm{H}}{ }^{9 \mathrm{~T}}\right)$ as functions of $d$, plotted as $\log$-log graphs (the red and blue lines are fitting results). (f) Temperature dependence of $R_{\mathrm{H}}{ }^{9 \mathrm{~T}}$ (red squares) and the saturation magnetization measured at $\mu_{0} H=7 \mathrm{~T}\left(M_{\mathrm{s}}{ }^{7 \mathrm{~T}}\right)$ (blue circles) for a film with $d=3.2 \mathrm{~nm}$. The black curves correspond to typical ferromagnetic and paramagnetic behaviors.

the background signal from the $c-\mathrm{Al}_{2} \mathrm{O}_{3}$ substrate (Fig. S5(a) [20]). Below approximately $30 \mathrm{~K}$, the $M_{\mathrm{s}}{ }^{7 \mathrm{~T}}$ gradually increased as the temperature was decreased, following the Bloch formula [23] that is typically applied to ferromagnets. In addition, the $M_{\mathrm{s}}{ }^{7 \mathrm{~T}}$ was independent of $d$ (Fig. S6 [20]), and therefore the magnetized volume in all the films was nearly constant. As shown in Fig. 2(f), both $R_{\mathrm{H}}{ }^{9 \mathrm{~T}}$ and $M_{\mathrm{s}}{ }^{7 \mathrm{~T}}$ exhibited similar temperature dependence. These observations indicated that the anomalous Hall effect (AHE) in the Pd-terminated ferromagnetic surface was responsible for the nonlinear $R_{\mathrm{H}}$.

According to the Stoner splitting model, surface magnetism should vanish when the surface polarity is cancelled.
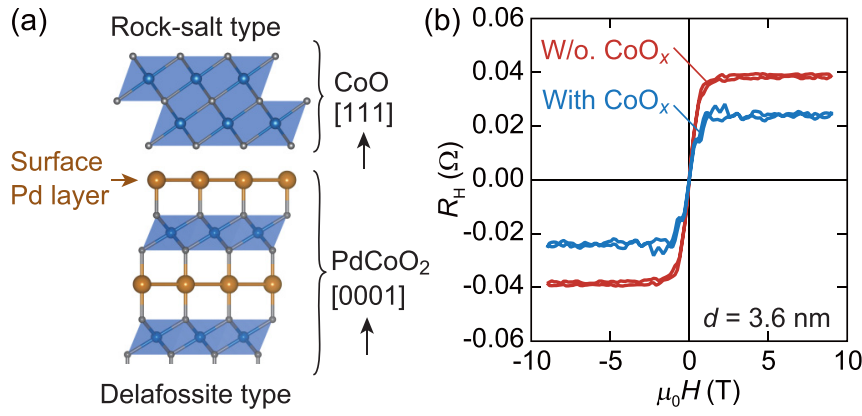

FIG. 3. Control of AHE by $\mathrm{CoO}_{x}$ capping. (a) Schematic illustration of the rock-salt type $\mathrm{CoO}$ (111) heterostructure and the delafossite-type $\mathrm{PdCoO}_{2}$ (0001). (b) $R_{\mathrm{H}}$ vs $\mu_{0} \mathrm{H}$ for $\mathrm{PdCoO}_{2}(3.6$ $\mathrm{nm})(\mathrm{red})$ and $\mathrm{CoO}_{x}(5 \mathrm{~nm}) / \mathrm{PdCoO}_{2}(3.6 \mathrm{~nm})$ (blue)

Taking advantage of the thin-film growth technique, we attempted to modulate the surface polarity by over layer deposition using the (111) surface of rock-salt-type $\mathrm{CoO}$, a single layer of which mimics the $\left[\mathrm{CoO}_{2}\right]^{-}$layer in $\mathrm{PdCoO}_{2}$ [Fig. 3(a)]. After the growth of $\mathrm{PdCoO}_{2}$, a 5-nm-thick $\mathrm{CoO}_{x}$ layer was deposited. The growth conditions used were identical to those used for $\mathrm{PdCoO}_{2}$ growth to minimize any influence on the $\mathrm{PdCoO}_{2}$ film quality during the $\mathrm{CoO}_{x}$ deposition. Although the exact phase of the thin $\mathrm{CoO}_{x}$ capping layer was not identified from the x-ray diffraction measurements, we consider the $\mathrm{CoO}$ (111) / $\mathrm{PdCoO}_{2}$ (0001) heterointerface to be the ideal model for the $\mathrm{CoO}_{x}$-capped $\mathrm{PdCoO}_{2}$ [Fig. 3(a)]. Capping with $\mathrm{CoO}_{x}$ drastically reduced the $R_{\mathrm{H}}$ by nearly $45 \%$ of the original value, while maintaining the saturation field [Fig. 3(b)]. The remaining finite $R_{\mathrm{H}}$ may have been caused by an electronic reconstruction that was required to satisfy charge neutrality throughout the entire $\mathrm{PdCoO}_{2}$ layer [20]. We note that accurate measurement of magnetization of $\mathrm{CoO}_{x}$-capped $\mathrm{PdCoO}_{2}$ surfaces was difficult because of finite magnetic signals from the $\mathrm{CoO}_{x}$ capping layers. Although further characterization is needed for quantitative discussions, this result underpins the Stoner origin of the observed AHE.

Comparison of the $R_{\mathrm{H}}$ and magnetization $(M)$ data highlighted a discrepancy in their behavior at low fields (Figs. S5(a) and S5(b) [20]). Changes in the measured $R_{\mathrm{H}}$ and the magnetization-driven anomalous Hall (AH) resistance with temperature are shown in Fig. 4(a). The $R_{\mathrm{AH}}-\mu_{0} H$ curve (red) was calculated from the $M-\mu_{0} H$, by assuming that $R_{\mathrm{AH}} \propto M$. As highlighted in blue, their difference (i.e., $R_{\mathrm{UH}}=R_{\mathrm{H}}-R_{\mathrm{AH}}$ ) became significant as the temperature was lowered. When calculating $R_{\mathrm{UH}}$, we assumed that the $R_{\mathrm{H}}$ at $7 \mathrm{~T}$ was dominated by the magnetization-driven $R_{\mathrm{AH}}$ because the high-field saturation values of $R_{\mathrm{AH}}$ and $M$ as functions of temperature overlapped [Fig. 2(f)]. This assumption corresponds to the situation in which the spins are parallel to the magnetic field at $7 \mathrm{~T}$. The $R_{\mathrm{UH}}$ (peak value) and $R_{\mathrm{AH}}$ each exhibited a distinct temperature dependence, which indicated that they had different origins [Fig. 4(b)]. The map of $R_{\mathrm{UH}}$ in the temperature- $\mu_{0} H$ plane [Fig. 4(c)] revealed a domelike structure that peaked at low field $\left(\mu_{0} H<3 \mathrm{~T}\right)$. As mentioned previously, the $d^{-2}$ dependence of $R_{\mathrm{UH}}$ indicated that $R_{\mathrm{UH}}$ originated on the surface (Fig. S7 [20]). 

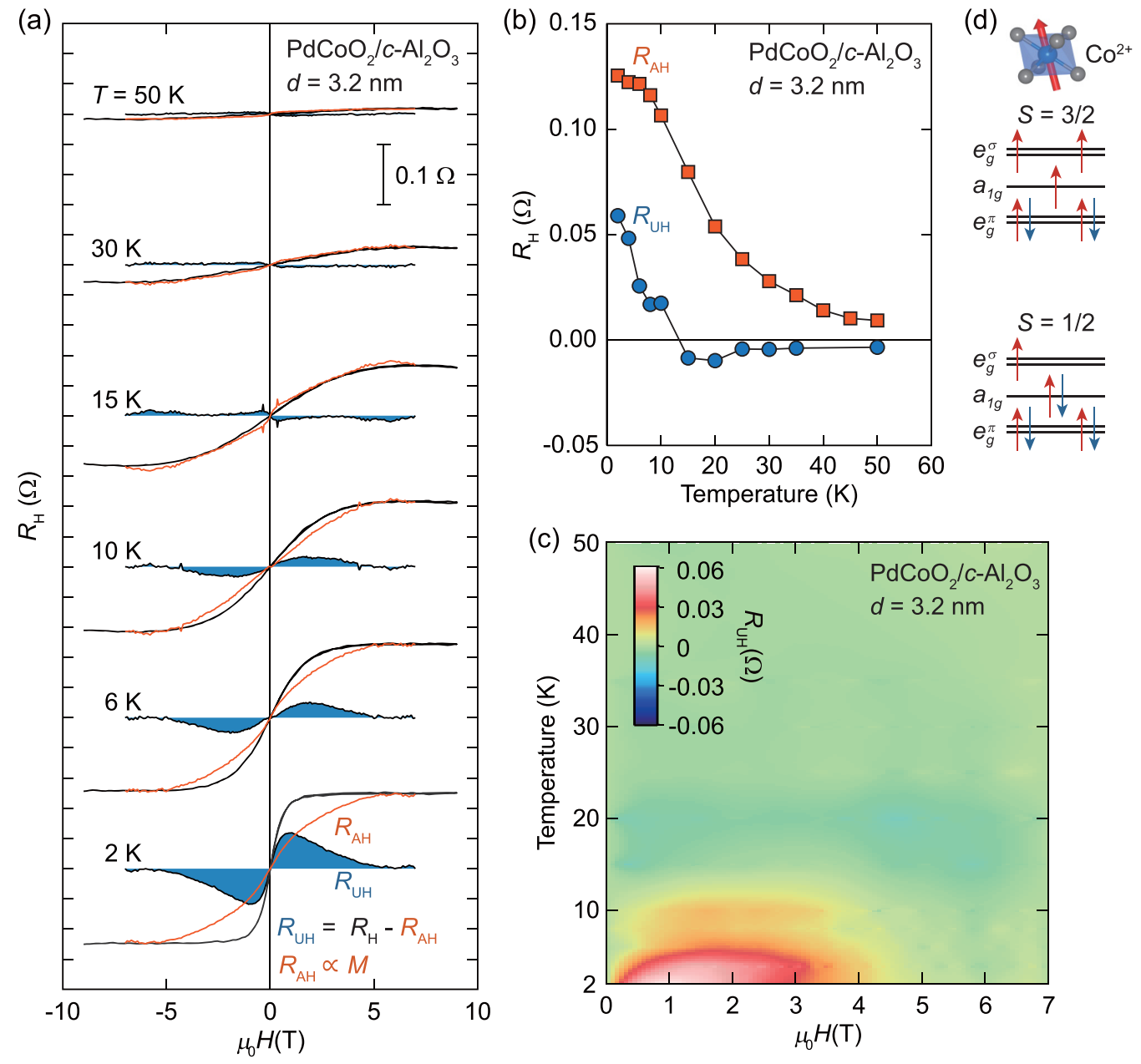

FIG. 4. Unconventional $\mathrm{AHE}$ of $\mathrm{PdCoO}_{2}$ thin films. (a) Magnetic field $\left(\mu_{0} H\right)$ dependence of $R_{\mathrm{H}}, R_{\mathrm{AH}}$, and $R_{\mathrm{UH}}$ for $d=3.2 \mathrm{~nm}$ at various temperatures. $R_{\mathrm{AH}}$ was calculated using the magnetization curves. (b) Temperature dependence of $R_{\mathrm{AH}}$ (red squares) and $R_{\mathrm{UH}}$ (blue circles). (c) The $R_{\mathrm{UH}}$ mapped as a function of temperature and magnetic field. (d) The electron configurations of $\mathrm{Co}^{2+}$ in the high-spin (top) and in low-spin (bottom) states.

The origin of the $R_{\mathrm{UH}}$ is discussed by considering the role of the $\mathrm{CoO}_{2}$ layer near the surface. The characteristic peaks that were observed at low field strength (1-2 T) in the $R_{\mathrm{UH}}$ plots in Fig. 4(a) were similar to the unconventional AHE reported for systems with nonuniform spin textures [24-28]. Nonlinear Hall resistance occurs in antiferromagnetic $\mathrm{PdCrO}_{2}$, which is a structural analogue of $\mathrm{PdCoO}_{2}$, despite the antiferromagnetic magnetization versus $\mu_{0} H$ curve [26]. The spin-frustrated triangular $\mathrm{Cr}^{3+}$ lattice is thought to play a role via the noncolinear spin arrangement [26]. In nominal $\mathrm{PdCoO}_{2}$ that contains $\mathrm{Co}^{3+}$ ions in the nonmagnetic lowspin state [Fig. 1(c)] [9,29], this mechanism is not expected to occur. However, when extra electrons are partially doped into the $\left[\mathrm{CoO}_{2}\right]^{-}$layer, the resultant finite moments within the $\mathrm{Co}^{2+}$ ions [Fig. 4(d)] could locally form a similar noncolinear spin structure in the nonmagnetic matrix. In fact, the $M_{\mathrm{s}}{ }^{7 \mathrm{~T}}$ in a 3.2-nm-thick $\mathrm{PdCoO}_{2}$ film was $3.4 \times 10^{-5} \mathrm{emu} \mathrm{cm}^{-2}$, which corresponded to $2.6 \mu_{\mathrm{B}}$ per areal unit ( $\sqrt{3} a b / 2$, where $a$ and $b$ are lattice constants of $\mathrm{PdCoO}_{2}$ ), and therefore much larger than the value deduced from the ARPES data (i.e., $0.59 \mu_{\mathrm{B}}$ per areal unit) [18].
The valence states of the Co ions were examined by a synchrotron x-ray absorption spectroscopy (XAS). Figure 5(a) shows the Co- $L_{2,3}$ XAS spectra of $\mathrm{PdCoO}_{2}$ films measured at $300 \mathrm{~K}$ for two different thicknesses $d$ of $2.7 \mathrm{~nm}$ and 8.8 $\mathrm{nm}$, which are normalized by the maximum intensities of their $L_{3}$-peaks. The spectra of $\mathrm{LaCoO}_{3}$ and $\mathrm{CoO}$ are shown as references for the $\mathrm{Co}^{3+}$ and $\mathrm{Co}^{2+}$ states, respectively. At first glance, the spectral shapes of $\mathrm{PdCoO}_{2}$ films and the energy positions of the main peaks at $L_{3}$ edge centered around $780.2 \mathrm{eV}$ (indicated by a black triangle) appear similar to those of the $\mathrm{Co}^{3+}$ states of the $\mathrm{LaCoO}_{3}$. This indicates that the formal valence of $\mathrm{Co}$ ions in $\mathrm{PdCoO}_{2}$ films is mainly $3+$, which is consistent with the fact that $\mathrm{PdCoO}_{2}$ has an alternating stacking structure of the $\mathrm{Pd}^{+}$and $\left[\mathrm{CoO}_{2}\right]^{-}$layers. However, with closer inspection, the $\mathrm{Co}-L_{3}$ XAS spectra of the $\mathrm{PdCoO}_{2}$ films with both $d=2.7$ and $8.8 \mathrm{~nm}$ have additional $\mathrm{Co}^{2+}$ contributions, which correspond to the shoulder features centered at around 777.2, 778.4, and $778.9 \mathrm{eV}$ (indicated by green triangles), suggesting that extra electrons are doped into the $\left[\mathrm{CoO}_{2}\right]^{-}$layer. Furthermore, these intensities are larger for the thinner film ( $d=2.7 \mathrm{~nm}$, red line) than the 

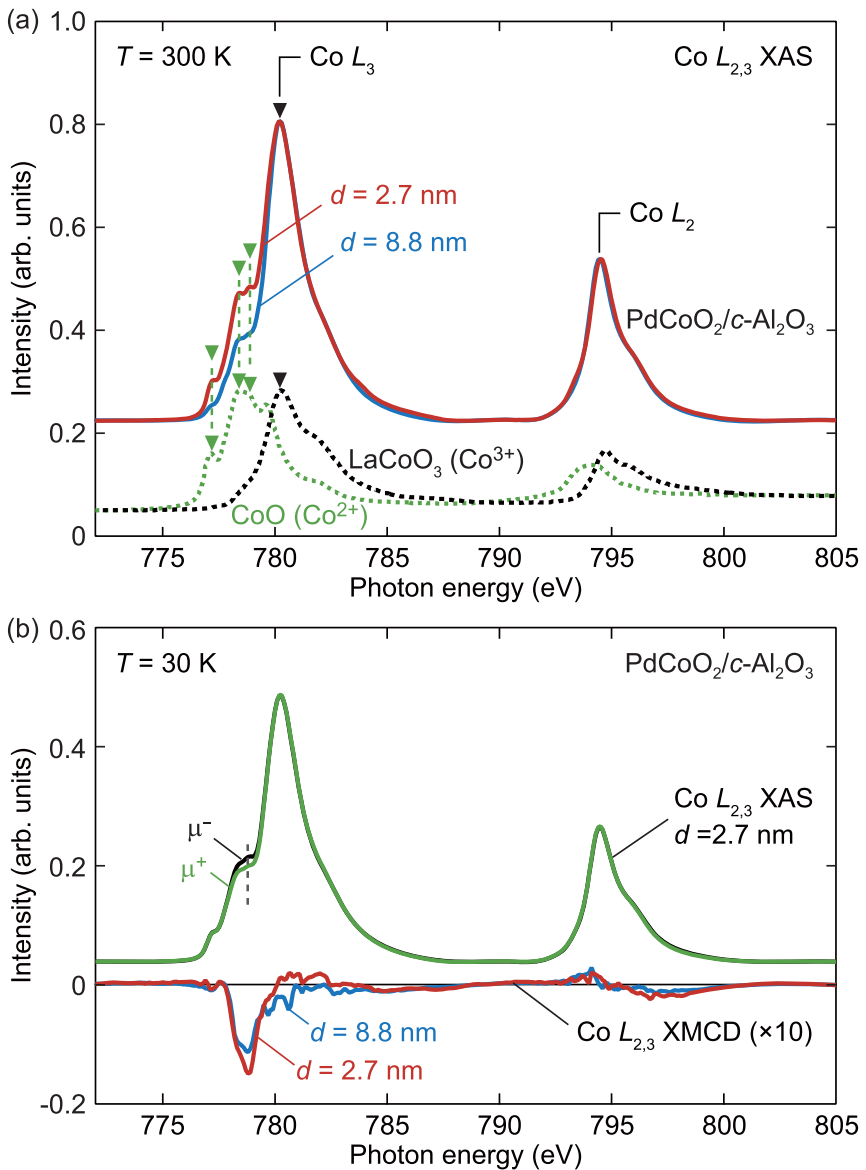

FIG. 5. Spectroscopic investigation of the $\mathrm{CoO}_{2}$ layers. (a) Co- $L_{2,3}$ XAS spectra of the $\mathrm{PdCoO}_{2}$ films with $d=2.7 \mathrm{~nm}$ (red line) and $8.8 \mathrm{~nm}$ (blue line) measured at $T=300 \mathrm{~K}$. The Co- $L_{2,3}$ XAS spectra of $\mathrm{LaCoO}_{3}$ (black dotted line) and $\mathrm{CoO}$ (green dotted line) are also shown as references for the $\mathrm{Co}^{3+}$ and $\mathrm{Co}^{2+}$ states, respectively [34]. The peak and shoulder structures that are attributed to the $\mathrm{Co}^{3+}$ and $\mathrm{Co}^{2+}$ states are indicated by black and green arrows, respectively. (b) XMCD data of the $\mathrm{PdCoO}_{2}$ thin films. Co- $L_{2,3}$ XAS spectra of the $\mathrm{PdCoO}_{2}$ film $(d=2.7 \mathrm{~nm})$ measured at $T=$ $30 \mathrm{~K}$, by positive $\left(\mu^{+}\right.$, green line) and negative $\left(\mu^{-}\right.$, black line) circular polarized lights, respectively. XMCD spectra $\left(\mu^{+}-\mu^{-}\right)$of the $\mathrm{PdCoO}_{2}$ films for $d=2.7$ (red line) and $8.8 \mathrm{~nm}$ (blue line) measured under the magnetic field of $5 \mathrm{~T}$ at $T=30 \mathrm{~K}$. The gray dashed line indicates the energy positions of the features characteristic to the $\mathrm{Co}^{2+}$ states. The spectra in (a) and (b) were offset for the easiness of comparison.

thicker one ( $d=8.8 \mathrm{~nm}$, blue line), indicating that the ratio of $\mathrm{Co}^{2+} / \mathrm{Co}^{3+}$ states increases with decreasing the thickness of a $\mathrm{PdCoO}_{2}$ film. Considering the surface (interface) sensitivity and element selectivity of XAS measurements, the evolution of $\mathrm{Co}^{2+}$ contributions in the thinner film means that extra $\mathrm{Co}^{2+}$ states mostly resides in the $\left[\mathrm{CoO}_{2}\right]^{-}$layer near the surface of the film.

To reveal the spin states of $\mathrm{Co}$ ions, we measured the circular dichroism of the XAS spectra for the $\mathrm{PdCoO}_{2}$ films. Figure 5(b) shows the Co- $L_{2,3} \mathrm{X}$-ray magnetic circular dichroism (XMCD) spectra of the $\mathrm{PdCoO}_{2}$ films with $d=2.7$ (red line) and $8.8 \mathrm{~nm}$ (blue line) measured at $T=30 \mathrm{~K}$. Clear XMCD signals were observed for the Co- $L_{2,3}$ edges of both $\mathrm{PdCoO}_{2}$ films, indicating that $\mathrm{Co}$ ions of $\mathrm{PdCoO}_{2}$ films have determinate magnetization. The peaks of the XMCD signals are located at almost the same energy position with that of shoulder structures in the XAS spectra which are derived from the $\mathrm{Co}^{2+}$ states [indicated by a gray dashed line in Fig. 5(b)]. The intensities of XMCD peaks decrease with increasing $d$. These results indicate that the magnetization emerges at $\mathrm{Co}^{2+}$ ions in the $\left[\mathrm{CoO}_{2}\right]^{-}$layer near the surface. We note that the $\mathrm{XMCD}$ measurements were performed at higher temperature than the transition temperature observed in the $R_{\mathrm{UH}}$ versus temperature curve shown in Fig. 4(b), because of the limitation of the XMCD apparatus. Thus, the observed magnetization of $\mathrm{Co}^{2+}$ ions were derived from the paramagneticlike states above the transition temperature. Nevertheless, existence of magnetic moments in the near-surface $\mathrm{CoO}_{2}$ layer explains the excess magnetization in SQUID magnetometry.

The near-surface $\mathrm{Co}^{2+}$ ions may have resulted from electron doping caused by band bending that extended over the insulating $\left[\mathrm{CoO}_{2}\right]^{-}$layer near the surface, although the role of oxygen vacancies must also be considered. Due to low density of mobile screening electrons in $\mathrm{CoO}_{2}$ layers [30], the energy bands of the $\mathrm{CoO}_{2}$ layer adjacent to the Pd surface layer could shift to higher binding energy, possibly changing the valence of $\mathrm{Co}$ ions from $3+$ to $2+$. In contrast to the three-dimensional, antiferromagnetic $\mathrm{PdCrO}_{2}$, the spins in the electron-doped $\mathrm{CoO}_{2}$ layer are likely to fluctuate because of the low dimensionality and could thus be easily aligned by an external magnetic field. Given the parallel spin alignment at high field strength, a $\mathrm{CoO}_{2}$ layer that is fully occupied with $\mathrm{Co}^{2+}$ ions corresponds to $3 \mu_{\mathrm{B}}$ per areal unit for the high-spin state [Fig. 4(d), top] and $1 \mu_{\mathrm{B}}$ per areal unit for the low-spin state [Fig. 4(d), bottom]. These values can account for the extra magnetization contribution of $\sim 2 \mu_{\mathrm{B}}$ per areal unit at $7 \mathrm{~T}$, which is added to the surface $\mathrm{Pd}$ contribution. The possible noncolinear magnetic moment in the electron-doped $\mathrm{CoO}_{2}$ layer could couple with the conduction electrons in the adjacent $\mathrm{Pd}$ layer, giving rise to the domelike $R_{\mathrm{UH}}$ in the temperature- $\mu_{0} H$ plane [Fig. 4(c)] that resembles the Hall resistance reported in noncolinear spin systems [24-28]. In particular, the $R_{\mathrm{UH}}$ showed behaviors in common with the unconventional $\mathrm{AHE}$ in $\mathrm{PdCrO}_{2}$ : sign reversal around $10 \mathrm{~K}$ and a negative peak that is broad along $\mu_{0} H$ around $20 \mathrm{~K}$ [26]. These features imply that the $R_{\mathrm{UH}}$ of $\mathrm{PdCoO}_{2}$ surface might be induced through similar mechanisms to those proposed for unconventional $\mathrm{AHE}$ in bulk $\mathrm{PdCrO}_{2}$ : Berry-phase [26,31] and Fermi-surface-reconstruction [32] mechanisms. In the former mechanism, unconventional $\mathrm{AHE}$ in $\mathrm{PdCrO}_{2}$ is explained by a fictitious magnetic field given by a nonzero phase gain of a conduction electron while it circuit around, assuming finite spin chirality on $\mathrm{Cr}$ sites [26]. In the $\mathrm{PdCoO}_{2}$ thin films, the broken inversion symmetry at the surface might contribute to induce finite $R_{\mathrm{UH}}$ via the Berry-phase mechanism. Regarding Fermi-surface-reconstruction mechanism for $\mathrm{PdCrO}_{2}$, coexistence of an electronlike Fermi surface and hole pockets due to magnetic ordering on $\mathrm{Cr}$ sites is important [32]. In the $\mathrm{PdCoO}_{2}$ surface, electronic reconstruction might occur 
due to the existence of electron-doped Co sites, which might make an intrinsic Berry-curvature mechanism relevant at low temperature. Further studies examining the detailed magnetic and electronic structures are needed to understand the causes of $R_{\mathrm{UH}}$ in $\mathrm{PdCoO}_{2}$ thin films.

In conclusion, we have studied the electronic properties at the polar surface of the layered, nonmagnetic metal $\mathrm{PdCoO}_{2}$ and have successfully detected surface ferromagnetism via the AHE using ultrathin $\mathrm{PdCoO}_{2}$ films. We have confirmed that the origin of the ferromagnetism was from the surface of the films by systematically varying the thickness and termination layer of the films. ARPES measurements revealed Stonersplit surface Pd bands at the Pd-terminated surface. The $R_{\mathrm{UH}}$ observed at low $\mu_{0} H$ is likely due to magnetic $\mathrm{Co}^{2+}$ ions near the surface. This work has demonstrated that using the thinfilm approach $[19,33]$ to study surface magnetism is highly effective because it drastically reduces bulk effects that can mask the surface properties. Revealing the $R_{\mathrm{UH}}$ mechanism requires an understanding of the detailed nature of surface ferromagnetism, especially the spin interactions of doped $\mathrm{Co}^{2+}$ ions and the role of broken inversion symmetry. The complex magnetic states at the surface, in combination with the hydrodynamic electron transport physics [13], make this layered oxide even more attractive for future research on spin-related transport phenomena.

\section{ACKNOWLEDGMENTS}

We thank T. Nakamura and H. Oinuma for their assistance in the ARPES experiments and K. Amemiya for supports of $\mathrm{X}$-ray magnetic circular dichroism measurements. This work is a cooperative program (Proposal No. 18G0407) of the CRDAM-IMR, Tohoku University. The work performed at KEK-PF was approved by the Program Advisory Committee (proposals 2018S2-004) at the IMSS, KEK. This work is partly supported by a Grant-in-Aid for Specially Promoted Research (No. 25000003), a Grant-in-Aid for Scientific Research on Innovative Areas (No. 15H05853), a Grant-in-Aid for Scientific Research (A) (Nos. 15H02022 and 16H02115), a Grant-in-Aid for Scientific Research (B) (No. 18H01821), a Grant-in-Aid for Scientific Research (C) (No. 16K05033), and a Grant-in-Aid for Early-Career Scientists (No. 18K14121 and 18K14130) from the Japan Society for the Promotion of Science (JSPS), JST CREST (JPMJCR18T2, JPMJCR18T1), Mayekawa Houonkai Foundation, and Tanaka Kikinzoku Memorial Foundation.
[1] B. Keimer, S. A. Kivelson, M. R. Norman, S. Uchida, and J. Zaanen, Nature (London) 518, 179 (2015).

[2] S. J. Moon et al., Phys. Rev. Lett. 101, 226402 (2008).

[3] Y. Maeno, H. Hashimoto, K. Yoshida, S. Nishizaki, T. Fujita, J. G. Bednorz, and F. Lichtenberg, Nature (London) 372, 532 (1994).

[4] K. Takada, H. Sakurai, E. Takayama-Muromachi, F. Izumi, R. A. Dilanian, and T. Sasaki, Nature (London) 422, 53 (2003).

[5] R. D. Shannon, D. B. Rogers, and C. T. Prewitt, Inorg. Chem. 10, 713 (1971).

[6] A. P. Mackenzie, Rep. Prog. Phys. 80, 032501 (2017).

[7] R. Daou, R. Frésard, V. Eyert, S. Hébert, and A. Maignan, Sci. Technol. Adv. Mater. 18, 919 (2017).

[8] R. Seshadri, C. Felser, K. Thieme, and W. Tremel, Chem. Mater. 10, 2189 (1998).

[9] M. Tanaka, M. Hasegawa, T. Higuchi, T. Tsukamoto, Y. Tezuka, S. Shin, and H. Takei, Physica B 245, 157 (1998).

[10] T. Higuchi, M. Hasegawa, M. Tanaka, H. Takei, S. Shin, and T. Tsukamoto, Jpn. J. Appl. Phys. 43, 699 (2004).

[11] V. Eyert, R. Frésard, and A. Maignan, Chem. Mater. 20, 2370 (2008).

[12] M. J. M. de Jong and L. W. Molenkamp, Phys. Rev. B 51, 13389 (1995).

[13] P. J. W. Moll, P. Kushwaha, N. Nandi, B. Schmidt, and A. P. Mackenzie, Science 351, 1061 (2016).

[14] J. Crossno et al., Science 351, 1058 (2016).

[15] D. A. Bandurin et al., Science 351, 1055 (2016).

[16] J. Gooth et al., Nat. Commun. 9, 4093 (2018).

[17] K. Kim, H. C. Choi, and B. I. Min, Phys. Rev. B 80, 035116 (2009).
[18] F. Mazzola et al., Proc. Natl. Acad. Sci. USA 115, 12956 (2018).

[19] T. Harada, K. Fujiwara, and A. Tsukazaki, APL Mater. 6, 046107 (2018).

[20] See Supplemental Material at http://link.aps.org/supplemental/ 10.1103/PhysRevResearch.2.013282 for detailed information.

[21] H.-J. Noh, J. Jeong, J. Jeong, E.-J. Cho, S. B. Kim, K. Kim, B I. Min, and H.-D. Kim, Phys. Rev. Lett. 102, 256404 (2009).

[22] V. Sunko et al., Nature (London) 549, 492 (2017).

[23] F. Bloch, Z. Phys. 61, 206 (1930).

[24] Y. Taguchi, Y. Oohara, H. Yoshizawa, N. Nagaosa, and Y. Tokura, Science 291, 2573 (2001).

[25] A. Neubauer, C. Pfleiderer, B. Binz, A. Rosch, R. Ritz, P. G. Niklowitz, and P. Böni, Phys. Rev. Lett. 102, 186602 (2009).

[26] H. Takatsu, S. Yonezawa, S. Fujimoto, and Y. Maeno, Phys. Rev. Lett. 105, 137201 (2010).

[27] J. Matsuno, N. Ogawa, K. Yasuda, F. Kagawa, W. Koshibae, N. Nagaosa, Y. Tokura, and M. Kawasaki, Sci. Adv. 2, e1600304 (2016).

[28] K. G. Rana et al., New J. Phys. 18, 085007 (2016).

[29] H.-J. Noh et al., Phys. Rev. B 80, 073104 (2009).

[30] L. Cheng, Q.-B. Yan, and M. Hu, Phys. Chem. Chem. Phys. 19, 21714 (2017).

[31] N. Nagaosa, J. Sinova, S. Onoda, A. H. MacDonald, and N. P. Ong, Rev. Mod. Phys. 82, 1539 (2010).

[32] J. M. Ok, Y. J. Jo, K. Kim, T. Shishidou, E. S. Choi, H.-J. Noh, T. Oguchi, B. I. Min, and J. S. Kim, Phys. Rev. Lett. 111 176405 (2013).

[33] M. Brahlek et al., Phys. Rev. Mater. 3, 093401 (2019).

[34] J.-H. Park, S.-W. Cheong, and C. T. Chen, Phys. Rev. B 55, 11072 (1997). 